\title{
Diffusion, Optimal Transport and Ricci Curvature for Metric Measure Spaces
}

Luigi Ambrosio (Scuola Normale Superiore, Pisa, Italy), Nicola Gigli (SISSA, Trieste, Italy) and Giuseppe Savaré (Università di Pavia, Italy)

Lower Ricci curvature bounds play a crucial role in several deep geometric and functional inequalities in Riemannian geometry and diffusion processes. Bakry-Émery [8] introduced an elegant and powerful technique, based on commutator estimates for differential operators and so-called $\Gamma$-calculus, to derive many sharp results. Their curvature-dimension condition has been further developed by many authors, mainly in the framework of Markov diffusion modelled on weighted Riemannian manifolds, with relevant applications to infinite dimensional problems.

A new synthetic approach relying on entropy and optimal transport has been more recently introduced by Lott, Sturm and Villani. It relies structurally on the notions of distance and measure and can therefore be used to extend the curvature-dimension condition to the general nonsmooth setting of metric measure spaces $(X, \mathrm{~d}, \mathrm{~m})$. Among its many beautiful properties, the synthetic approach is stable with respect to measured Gromov convergence.

The equivalence of the two points of view can be directly proved in a smooth differential setting but it is a difficult task in a general metric framework, when explicit calculations in local charts are hard (if not impossible) to justify.

We will try to give a brief and informal introduction to the two approaches and show how the Otto variational interpretation of the Fokker-Planck equation and the theory of metric gradient flows has provided a unifying point of view, which allows one to prove their equivalence for arbitrary metric measure spaces. As a byproduct, by combining $\Gamma$-calculus and optimal transport techniques, an impressive list of deep results in Riemannian geometry and smooth diffusion have a natural counterpart in the nonsmooth metric measure framework.

\section{Ricci curvature and $\Gamma$-calculus in a smooth setting}

In order to introduce and explain the basic notions we will deal with, we consider a smooth, complete and connected $d$ dimensional Riemannian manifold ( $\mathbb{M}, g$ ) endowed with its Riemannian distance $d_{g}$ and a reference measure $m=e^{-V} V_{o l}$ that is absolutely continuous with respect to the Riemannian volume form $\mathrm{Vol}_{\mathrm{g}}$; its density is associated to the smooth potential $V: \mathbb{M} \rightarrow \mathbb{R}$. The metric tensor $g$ induces a norm $|\nabla f|_{\mathrm{g}}$ of the gradient of a smooth function $f: \mathbb{M} \rightarrow \mathbb{R}$, given in local coordinates by $|\nabla f|_{\mathrm{g}}^{2}=\sum_{i, j} \mathrm{~g}^{i j} \partial_{i} f \partial_{j} f$.

The combination with the reference measure $m$ gives rise to the quadratic energy form

$$
\mathcal{E}(f):=\int_{\mathbb{M}}|\nabla f|_{\mathrm{g}}^{2} \mathrm{~d} \mathfrak{m}, \quad \mathcal{E}(f, g):=\int_{\mathbb{M}}\langle\nabla f, \nabla g\rangle_{\mathrm{g}} \mathrm{d} \mathfrak{m},
$$

and to the second order differential operator $\mathrm{L}=\Delta_{\mathrm{g}}-\langle\nabla V, \cdot\rangle_{\mathrm{g}}$ satisfying the integration-by-parts formula

$$
\mathcal{E}(f, g)=-\int_{\mathbb{M}} f \mathrm{~L} g \mathrm{~d} \mathfrak{m} .
$$

$\mathrm{L}$ reads in local coordinates as

$$
\mathrm{L} f=\mathrm{e}^{W} \sum_{i, j} \partial_{i}\left(\mathrm{e}^{-W} \mathrm{~g}^{i j} \partial_{j} f\right), \quad W=V+\frac{1}{2} \log \operatorname{det}\left(\mathrm{g}^{i j}\right),
$$

and generates a semigroup $\left(\mathrm{P}_{t}\right)_{t \geq 0}$ through the solution $f_{t}=$ $\mathrm{P}_{t} f$ of the diffusion equation

$$
\partial_{t} f_{t}=\mathrm{L} f_{t} \quad \text { in }[0, \infty) \times \mathbb{M}, \quad f_{0}=f .
$$

Whenever $f_{0} \in \mathrm{C}_{c}^{\infty}(\mathbb{M})$ is smooth with compact support, $f$ is a classical $\mathrm{C}^{\infty}$ solution to (2) and one can extend $\mathrm{L}$ from $\mathrm{C}_{c}^{\infty}(\mathbb{M})$ to a self-adjoint operator in $L^{2}(X, \mathrm{~m})$ generating a Markov semigroup.

Example 1. Choosing $\mathbb{M}=\mathbb{R}^{d}$ with the Euclidean metric and $V \equiv 0$, one gets the Laplace operator and the corresponding heat flow. More general choices of $V$ yield the drift-diffusion operator and the weighted Dirichlet form

$$
\mathrm{L} f=\Delta f-\nabla V \cdot \nabla f, \quad \mathcal{E}(f):=\int_{\mathbb{R}^{d}}|\nabla f|^{2} \mathrm{e}^{-V} \mathrm{~d} x
$$

the Gaussian space $\left(\mathbb{R}^{d},|\cdot|, \mathfrak{m}_{G}\right)$ corresponds to the Gaussian measure $\mathfrak{m}_{G}$ associated to $V_{G}(x):=\frac{1}{2}|x|^{2}+\frac{d}{2} \ln (2 \pi)$ and to the Ornstein-Uhlenbeck operator $\mathrm{L} f=\Delta f-x \cdot \nabla f$.

The usual elliptic second order operators in divergence form

$$
\mathrm{L} f=\sum_{i, j} \partial_{i}\left(\mathrm{~g}^{i j} \partial_{j} f\right), \quad \mathcal{E}(f)=\int_{\mathbb{R}^{d}} \sum_{i, j} \mathrm{~g}^{i j} \partial_{i} f \partial_{j} f \mathrm{~d} x,
$$

are associated to the uniformly elliptic metric tensor $\mathrm{g}^{i j}$ and to the potential $V:=-\frac{1}{2} \log \operatorname{det}\left(\mathrm{g}^{i j}\right)$ by the expression of the volume measure in local coordinates.

The Laplace-Beltrami operator $\Delta_{\mathrm{g}}$ and the energy form

$$
\mathrm{L} f=\Delta_{\mathrm{g}} f, \quad \mathcal{E}(f)=\int_{\mathbb{M}}|\nabla f|_{\mathrm{g}}^{2} \mathrm{dVol}_{\mathrm{g}}
$$

in a Riemannian manifold corresponds to the choice $V \equiv 0$. 
It is interesting to note that the operator $\mathrm{L}$ encodes the information concerning the metric tensor, which can be reconstructed by the commutation identity yielding the $\Gamma$-tensor

$$
\Gamma(f, g):=\frac{1}{2}(\mathrm{~L}(f g)-(\mathrm{L} f) g-f \mathrm{~L} g), \quad \Gamma(f):=\Gamma(f, f),
$$

since one easily gets

$$
\Gamma(f, g)=\langle\nabla f, \nabla g\rangle_{\mathrm{g}}, \quad \Gamma(f)=|\nabla f|_{\mathrm{g}}^{2}
$$

$\Gamma$ also characterises the class of the Lipschitz functions (and thus the Riemannian distance), since

$$
\Gamma(f) \leq L^{2} \quad \Rightarrow \quad f \text { is } L \text {-Lipschitz. }
$$

Bakry-Émery [8, 9] introduced a further geometric tensor, called $\Gamma_{2}$, obtained by an iterated commutation:

$$
\Gamma_{2}(f):=\frac{1}{2} \mathrm{~L} \Gamma(f)-\Gamma(f, \mathrm{~L} f) .
$$

Thanks to the Bochner-Lichnerowicz identity, the $\Gamma_{2}$ tensor can be expressed by the following remarkable formula, involving, in a crucial way, Ricci curvature and the Hessian of $V$ :

$$
\Gamma_{2}(f)=\left|\nabla^{2} f\right|_{\mathrm{g}}^{2}+\operatorname{Ric}_{\mathrm{g}}(\nabla f, \nabla f)+\nabla_{\mathrm{g}}^{2} V(\nabla f, \nabla f) .
$$

According to Barky-Émery, the weighted Riemannian manifold $\left(\mathbb{M}, \mathrm{d}_{\mathrm{g}}, \mathrm{m}\right)$ satisfies the curvature-dimension condition $\mathrm{BE}(K, N), K \in \mathbb{R}, N \in[1, \infty]$ if, for every smooth function $f$,

$$
\Gamma_{2}(f) \geq K \Gamma(f)+\frac{1}{N}|\mathrm{~L} f|^{2} .
$$

Example 2. When $V \equiv 0$, the $\operatorname{BE}(K, N)$ condition is equivalent to $\operatorname{Ric}_{\mathrm{g}} \geq K \mathrm{~g}$ and $d \leq N$. In particular, Euclidean space $\mathbb{R}^{d}$ satisfies the $\operatorname{BE}(0, d)$ condition; the $d$-dimensional unit sphere $\mathbb{S}^{d}$ (resp. hyperbolic space $\mathbb{H}^{d}$ ) is the reference model for $\operatorname{BE}(d-1, d)($ resp. $\operatorname{BE}(-(d-1), d))$.

When a general potential $V$ is involved, (5) also reflects the convexity of $V$ : in the simplest Euclidean case of $\mathbb{R}^{d}$ and $N=\infty,(5)$ is equivalent to $\nabla^{2} V \geq K I$. In particular, Gaussian space $\left(\mathbb{R}^{d},|\cdot|, \mathrm{m}_{G}\right)$ satisfies the $\operatorname{BE}(1, \infty)$ condition.

\section{Two equivalent formulations of the curvature-dimension condition}

The $\operatorname{BE}(K, N)$ condition has many deep and beautiful functional and geometric consequences (some of them are listed at the end of this paper in Section 7). Here we focus on two relevant (and seemingly far-reaching) aspects.

\section{Pointwise gradient estimates for Markov diffusion}

A first application concerns the behaviour of the semigroup $\left(\mathrm{P}_{t}\right)_{t \geq 0}$ associated to (2).

Theorem 3 ([8, 45]). The weighted manifold $\left(\mathbb{M}, d_{g}, m\right)$ satisfies the curvature-dimension condition $\mathrm{BE}(K, N)$ if and only if, for every smooth function $f$ with compact support and for every $t \geq 0$,

$$
\mathrm{e}^{2 K t}\left|\nabla \mathrm{P}_{t} f\right|_{\mathrm{g}}^{2}+\frac{2}{N} E_{2 K}(t)\left|\mathrm{LP}_{t} f\right|^{2} \leq \mathrm{P}_{t}|\nabla f|_{\mathrm{g}}^{2}
$$

where

$$
E_{\lambda}(t):=\int_{0}^{t} \mathrm{e}^{\lambda s} \mathrm{~d} s= \begin{cases}\lambda^{-1}\left(\mathrm{e}^{\lambda t}-1\right) & \text { if } \lambda \neq 0 \\ t & \text { if } \lambda=0 .\end{cases}
$$

In the flat Euclidean case $\mathbb{M}=\mathbb{R}^{d}, V \equiv 0,(6)$ with $N=\infty$ simply follows by Jensen's inequality and the commutation property of the heat equation $\nabla \mathrm{P}_{t} f=\mathrm{P}_{t}(\nabla f)$. In the general case, (6) reflects the commutator bounds coded in (5); the simplest situation is provided by the $\operatorname{BE}(K, \infty)$ case, when (6) follows (at least formally - see, for example, [9, Sec. 3.2.3]) by the monotonicity property of the quantity

$$
\Lambda(s):=\mathrm{P}_{s}\left(\Gamma\left(\mathrm{P}_{t-s} f\right)\right), \quad 0 \leq s \leq t
$$

which satisfies the differential inequality

$$
\frac{\mathrm{d}}{\mathrm{d} s} \Lambda(s)=2 \mathrm{P}_{s}\left(\Gamma_{2}\left(\mathrm{P}_{t-s} f\right)\right) \geq 2 K \mathrm{P}_{s}\left(\Gamma\left(\mathrm{P}_{t-s} f\right)\right)=2 K \Lambda(s) .
$$

Brunn-Minkowski and Prékopa-Leindler inequalities

A second instance of application of curvature bounds concerns the curved version of the celebrated Brunn-Minkowski inequality

$$
\operatorname{Vol}((1-\vartheta) A+\vartheta B) \geq(\operatorname{Vol}(A))^{1-\vartheta}(\operatorname{Vol}(B))^{\vartheta}, \quad \vartheta \in[0,1]
$$

for an arbitrary couple of Borel sets $A, B \subset \mathbb{R}^{d}$; here, (1 $\vartheta) A+\vartheta B=\{(1-\vartheta) a+\vartheta b: a \in A, b \in B\}$.

In order to state it in a Riemannian manifold, it is convenient to denote by $Z_{\vartheta}(a, b), a, b \in \mathbb{M}$, the set of interpolating points $x \in \mathbb{M}$ satisfying

$$
\mathrm{d}_{\mathrm{g}}(a, x)=(1-\vartheta) \mathrm{d}_{\mathrm{g}}(a, b), \mathrm{d}_{\mathrm{g}}(x, b)=\vartheta \mathrm{d}_{\mathrm{g}}(a, b), \quad \vartheta \in[0,1]
$$

Theorem 4 ([17]). The weighted manifold $\left(\mathbb{M}, d_{g}, m\right)$ satisfies the curvature-dimension condition $\operatorname{BE}(K, \infty)$ if and only if, for every $\vartheta \in[0,1]$ and Borel functions $f, f_{0}, f_{1}: \mathbb{M} \rightarrow$ $[0, \infty)$ satisfying

$$
f(x) \geq \exp \left(-\frac{K}{2} \vartheta(1-\vartheta) \mathrm{d}_{\mathrm{g}}^{2}(a, b)\right) f_{0}(a)^{1-\vartheta} f_{1}(b)^{\vartheta}
$$

whenever $a, b \in \mathbb{M}$ and $x \in Z_{\vartheta}(a, b)$, it holds that

$$
\int_{\mathbb{M}} f \mathrm{~d} \mathfrak{m} \geq\left(\int_{\mathbb{M}} f_{0} \mathrm{~d} \mathfrak{m}\right)^{1-\vartheta}\left(\int_{\mathbb{M}} f_{1} \mathrm{~d} \mathrm{~m}\right)^{\vartheta} .
$$

In particular, if $K \geq 0$, we have

$$
m\left(Z_{\vartheta}(A, B)\right) \geq(m(A))^{1-\vartheta}(m(B))^{\vartheta},
$$

where

$$
Z_{\vartheta}(A, B):=\left\{x \in Z_{\vartheta}(a, b) \text { for some } a \in A, b \in B\right\} .
$$


(8) admits the refined $N$-dimensional version,

$$
\mathfrak{m}\left(Z_{\vartheta}(A, B)\right)^{1 / N} \geq \tau_{K, N}^{1-\vartheta}(\delta) \mathfrak{m}(A)^{1 / N}+\tau_{K, N}^{\vartheta}(\delta) \mathfrak{m}(B)^{1 / N},
$$

where $\tau_{K, N}(\cdot)$ are suitable distortion coefficients only depending on $K$ and $N$ and $\delta$ is the minimal (resp. maximal) distance between the points of $A$ and $B$ if $K \geq 0$ (resp. $K<0$ ).

We shall see that the optimal transport point of view provides a nice unifying interpretation of (6) and (7a,b), which will only depend on the metric $d_{g}$ and on the reference measure $m$ defined on $\mathbb{M}$, without referring to its differential structure. The basic idea is to lift the geometric properties of $\mathbb{M}$ to the space of Borel probability measures $\mathcal{P}(\mathbb{M})$ : (2) can be interpreted as a gradient flow in $\mathcal{P}(\mathbb{M})$ and an interpolation of sets as in (8) becomes a geodesic in $\mathcal{P}(\mathbb{M})$. In both cases, the curvature-dimension condition can be characterised by the behaviour of the entropy functional along these two classes of curves.

\section{Optimal transport and the geometry of probability measures}

Optimal transport provides a natural way to introduce a geometric distance between probability measures, which reflects the properties of $d_{g}$ in $\mathbb{M}$. We introduce it in the more general framework of a complete and separable metric space $(X, \mathrm{~d})$.

We call $\mathcal{P}_{2}(X)$ the space of Borel probability measures with finite quadratic moment: every $\mu \in \mathcal{P}_{2}(X)$ satisfies

$$
\int_{X} d^{2}\left(x, x_{o}\right) d \mu(x)<\infty
$$

for some (and thus any) reference point $x_{o} \in X$.

For a given couple of measures $\mu_{0}, \mu_{1} \in \mathcal{P}_{2}(X)$, we consider the collection Plan $\left(\mu_{0}, \mu_{1}\right)$ of all the transport plans or couplings between $\mu_{0}, \mu_{1}$, i.e. measures $\mu \in \mathcal{P}(X \times X)$ with marginals $\mu_{0}, \mu_{1}$, thus satisfying

$$
\boldsymbol{\mu}(A \times X)=\mu_{0}(A), \quad \boldsymbol{\mu}(X \times B)=\mu_{1}(B)
$$

for every Borel subset $A, B \subset X$. The squared KantorovichRubinstein-Wasserstein distance $\mathrm{W}_{\mathrm{d}}\left(\mu_{0}, \mu_{1}\right)$ (Wasserstein distance, for short) is then defined as

$$
\mathrm{W}_{\mathrm{d}}^{2}\left(\mu_{0}, \mu_{1}\right):=\min _{\boldsymbol{\mu} \in \operatorname{Plan}\left(\mu_{0}, \mu_{1}\right)} \int_{X \times X} \mathrm{~d}^{2}\left(x_{0}, x_{1}\right) \mathrm{d} \boldsymbol{\mu}\left(x_{0}, x_{1}\right) .
$$

Equation (9) is an important example of the class of optimal transport problems, where the squared distance function $\mathrm{d}^{2}\left(x_{0}, x_{1}\right)$ in (9) is replaced by a general cost c $: X \times X \rightarrow \mathbb{R}$.

$\mathrm{W}_{\mathrm{d}}$ is a distance on $\mathcal{P}_{2}(X)$ inducing the topology of weak convergence with quadratic moments, i.e. convergence of all the integrals $\mu \mapsto \int_{X} \phi \mathrm{d} \mu$ whenever $\phi: X \rightarrow \mathbb{R}$ is continuous with at most quadratic growth. $\left(\mathcal{P}_{2}(X), \mathrm{W}_{\mathrm{d}}\right)$ is a complete and separable metric space and it inherits other useful properties from $(X, \mathrm{~d})$ such as compactness or existence of geodesics. We refer the interested reader to a number of books $[3,44,39]$.

\section{The dynamical formulation of Benamou-Brenier}

The Wasserstein distance $\mathrm{W}_{\mathrm{d}}$ enjoys two other important $d y$ namical characterisations, which play a crucial role in the geometric formulation of the properties discussed in Section 2.

According to the first one, which is due to BenamouBrenier [10], the Wasserstein distance between $\mu_{0}, \mu_{1} \in$ $\mathcal{P}_{2}(\mathbb{M})$ in the Riemannian manifold $\mathbb{M}$ can be evaluated by minimising the action

$$
\mathrm{W}_{\mathrm{d}}^{2}\left(\mu_{0}, \mu_{1}\right)=\min \int_{0}^{1} \int_{\mathbb{M}}|\boldsymbol{v}|_{\mathrm{g}}^{2} \mathrm{~d} \mu_{t} \mathrm{~d} t
$$

among all the weakly continuous solutions $(\mu, v)$ of the continuity equation

$$
\partial_{t} \mu_{t}+\operatorname{div}_{\mathrm{g}}\left(\mu_{t} \boldsymbol{v}_{t}\right)=0, \quad t \in[0,1]
$$

connecting $\mu_{0}$ to $\mu_{1}$. Equation (10b) has to be intended in duality with smooth test functions, i.e.

$$
\int_{0}^{1} \int_{\mathbb{M}}\left(\partial_{t} \zeta(x, t)+\langle\nabla \zeta(x, t), \boldsymbol{v}(x, t)\rangle_{\mathrm{g}}\right) \mathrm{d} \mu_{t} \mathrm{~d} t=0
$$

for every $\zeta \in \mathrm{C}_{c}^{\infty}(\mathbb{M} \times(0,1))$.

The Benamou-Brenier representation $(10 \mathrm{a}, \mathrm{b})$ can be further extended to Lipschitz (or even absolutely continuous) curves $\left(\mu_{t}\right)_{t \in[0,1]}$ in $\mathcal{P}_{2}(\mathbb{M})$ : they can be characterised as solutions to the continuity equation (10b) with a Borel vector field $\boldsymbol{v}$ satisfying

$$
\int_{X}\left|\boldsymbol{v}_{t}(x)\right|_{\mathrm{g}}^{2} \mathrm{~d} \mu_{t}(x)=\lim _{h \rightarrow 0} \frac{\mathrm{W}_{\mathrm{d}}^{2}\left(\mu_{t}, \mu_{t+h}\right)}{h^{2}}
$$

for a.e. $t \in(0,1)$. The limit on the right side of equation (11) has a natural interpretation as the squared metric velocity $\left|\dot{\mu}_{t}\right|_{\mathrm{W}_{\mathrm{d}}}^{2}$ of $\mu$ at the time $t$, whose integral

$$
\int_{0}^{1}\left|\dot{\mu}_{t}\right|_{\mathrm{W}_{\mathrm{d}}} \mathrm{d} t=\int_{0}^{1}\left(\int_{\mathbb{M}}\left|\boldsymbol{v}_{t}\right|_{\mathrm{g}}^{2} \mathrm{~d} \mu_{t}\right)^{1 / 2} \mathrm{~d} t
$$

expresses the length of the curve in $\mathcal{P}_{2}(X)$.

\section{Duality and Hamilton-Jacobi equations}

The second characterisation of $\mathrm{W}_{\mathrm{d}}$ is a dynamical version of the dual Kantorovich formulation

$$
\frac{1}{2} \mathrm{~W}_{\mathrm{d}}^{2}\left(\mu_{0}, \mu_{1}\right)=\sup _{\phi \in \mathrm{C}_{b}(X)} \int_{X} \mathrm{Q}_{1} \phi(y) \mathrm{d} \mu_{1}(x)-\int_{X} \phi(x) \mathrm{d} \mu_{0}(x)
$$

shared with all optimal transport problems. Here, $Q_{1}$ denotes the inf-convolution

$$
\mathrm{Q}_{1} \phi(y):=\inf _{x \in X} \frac{1}{2} \mathrm{~d}^{2}(x, y)+\phi(x)
$$

and it is the value at time $t=1$ of the Hopf-Lax evolution $\left(Q_{t}\right)_{t \geq 0}$, defined by

$$
\mathrm{Q}_{t} \phi(y):=\inf _{x \in X} \frac{1}{2 t} \mathrm{~d}^{2}(x, y)+\phi(x), \quad \phi \in \mathrm{C}_{b}(X) .
$$


When $(X, \mathrm{~d})$ is a length space, i.e. for every couple of points $x, y \in X$ and $\varepsilon>0$, there exists an $\varepsilon$-midpoint $z_{\varepsilon} \in X$ satisfying

$$
\max \left(\mathrm{d}\left(x, z_{\varepsilon}\right), \mathrm{d}\left(z_{\varepsilon}, y\right)\right) \leq(1 / 2+\varepsilon) \mathrm{d}(x, y),
$$

then $\left(Q_{t}\right)_{t \geq 0}$ is a semigroup in $\operatorname{Lip}_{b}(X)$, the space of bounded and Lipschitz real functions defined in $X$. In the Euclidean case $X=\mathbb{R}^{d}, \phi_{t}(\cdot):=\mathrm{Q}_{t} \phi$ is the unique viscosity solution of the Hamilton-Jacobi equation

$$
\partial_{t} \phi_{t}(x)+\frac{1}{2}\left|\nabla \phi_{t}(x)\right|^{2}=0, \quad \lim _{t \downarrow 0} \phi_{t}(x)=\phi(x),
$$

and an analogous property holds in the Riemannian setting. In general metric spaces, one can give a metric interpretation to the quantity $|\nabla \phi|$ by considering the local slope or local Lipschitz constant of a map $\phi: X \rightarrow \mathbb{R}$,

$$
|\nabla \phi|(x):=\limsup _{y \rightarrow x} \frac{|\phi(y)-\phi(x)|}{\mathrm{d}(y, x)} .
$$

It is possible to prove [4] that, for every $x \in X, \phi_{t}(\cdot):=\mathrm{Q}_{t} \phi$ satisfies

$$
\partial_{t} \phi_{t}(x)+\frac{1}{2}\left|\nabla \phi_{t}\right|^{2}(x)=0
$$

for every $t \in(0, \infty)$ with at most countably many exceptions. A further regularisation in time [2] shows that

$$
\frac{1}{2} \mathrm{~W}_{\mathrm{d}}^{2}\left(\mu_{0}, \mu_{1}\right)=\sup \int_{X} \phi_{1}(y) \mathrm{d} \mu_{1}(y)-\int_{X} \phi_{0}(x) \mathrm{d} \mu_{0}(x),
$$

where the supremum runs among all the regular subsolutions $\phi \in \mathrm{C}^{1}\left([0,1]\right.$; $\left.\operatorname{Lip}_{b}(X)\right)$ of (13), i.e. solving

$$
\partial_{t} \phi_{t}+\frac{1}{2}\left|\nabla \phi_{t}\right|^{2}(x) \leq 0 \quad \text { in } X \times[0,1]
$$

\section{$4 \quad$ Wasserstein distance and lower curvature bounds in smooth Riemannian manifolds}

The two equivalent formulations of the curvature-dimension condition $\operatorname{BE}(K, \infty)$ in Section 2 have nice counterparts in terms of the Kantorovich-Rubinstein-Wasserstein distance. In order to keep the exposition simpler, we just focus on the case $N=\infty$.

\section{Pointwise gradient estimates and Wasserstein contraction}

The first formulation relies on the Kuwada duality result [34], which exploits the dual dynamic representation formula (14a,b) and deals with a couple of dual maps: $\mathrm{P}: \mathrm{C}_{b}(X) \rightarrow$ $\mathrm{C}_{b}(X)$ linear and continuous and $\mathrm{P}^{*}: \mathcal{P}(X) \rightarrow \mathcal{P}(X)$ satisfying

$$
\int_{X} \mathrm{P} \phi \mathrm{d} \mu=\int_{X} \phi \mathrm{d}\left(\mathrm{P}^{*} \mu\right) \quad \text { for every } \varphi \in \mathrm{C}_{b}(X), \mu \in \mathcal{P}(X) .
$$

Kuwada's duality states that in length metric spaces and under minimal assumptions, $P$ preserves Lipschitz functions and satisfies the pointwise bound

$$
|\nabla \mathrm{P} \phi| \leq L \mathrm{P}|\nabla \phi| \quad \text { for every } \phi \in \operatorname{Lip}_{b}(X)
$$

if and only if $\mathrm{P}^{*}$ is a $L$-Wasserstein contraction, i.e.

$$
\mathrm{W}_{\mathrm{d}}\left(\mathrm{P}^{*} \mu, \mathrm{P}^{*} v\right) \leq L \mathrm{~W}_{\mathrm{d}}(\mu, v) \text { for every } \mu, v \in \mathcal{P}_{2}(X) \text {. }
$$

In the case of the Markov semigroup $\left(\mathrm{P}_{t}\right)_{t \geq 0}$ introduced in Section 1 in a Riemannian setting, one gets the following theorem [38, 42].

Theorem 5. The semigroup $\left(\mathrm{P}_{t}\right)_{t \geq 0}$ satisfies the pointwise bound (6) of Theorem 3 with $K \in \mathbb{R}, N=\infty$, if and only if the semigroup $\left(\mathrm{P}_{t}^{*}\right)_{t \geq 0}$, defined on $\mathrm{m}$-absolutely continuous measures $\mu=\varrho$ by $\mathrm{P}_{t}^{*} \mu:=\left(\mathrm{P}_{t} \varrho\right) \mathrm{m}$, admits a unique extension to $\mathcal{P}_{2}(X)$ satisfying the contraction property

$$
\mathrm{W}_{\mathrm{d}}\left(\mathrm{P}_{t}^{*} \mu, \mathrm{P}_{t}^{*} v\right) \leq \mathrm{e}^{-K t} \mathrm{~W}_{\mathrm{d}}(\mu, v) \quad \text { for every } \mu, v \in \mathcal{P}_{2}(X) \text {. }
$$

Even more refined estimates hold in the case $N<\infty$.

Notice that one of the advantages of (15) with respect to (6) and to the differential formulation of the $\operatorname{BE}(K, \infty)$ condition given by (5) relies on the weaker regularity requirement of its formulation: it involves probability measures and the Markov semigroup $\left(\mathrm{P}_{t}\right)_{t>0}$ but avoids local differentiability structures. This is one of the recurrent themes of pushing the geometric information coded into lower Ricci curvature bounds toward general metric measure spaces.

\section{Prékopa-Leindler inequality and convexity of the entropy}

A second crucial interpretation provides a link between the weighted Prékopa-Leindler inequality $(7 \mathrm{a}, \mathrm{b})$, the geometric notion of geodesics in metric spaces and the entropy functional.

Geodesics in a metric space $\left(X, \mathrm{~d}_{X}\right)$ are length-minimising curves $\left(\mathrm{x}_{\vartheta}\right)_{\vartheta \in[0,1]}$ in $X$ satisfying

$$
\mathrm{d}_{X}\left(\mathrm{x}_{\vartheta_{0}}, \mathrm{x}_{\vartheta_{1}}\right)=\left(\vartheta_{1}-\vartheta_{0}\right) \mathrm{d}_{\mathcal{X}}\left(\mathrm{x}_{0}, \mathrm{x}_{1}\right), \quad 0 \leq \vartheta_{0} \leq \vartheta_{1} \leq 1 .
$$

A real functional $\Phi: D(\Phi) \subset \mathcal{X} \rightarrow \mathbb{R}$ is called geodesically $K$-convex if every couple of points $x_{0}, x_{1} \in D(\Phi)$ can be connected by a geodesic $\left(\mathrm{x}_{\vartheta}\right)_{\vartheta \in[0,1]}$ along which

$$
\Phi\left(\mathrm{x}_{\vartheta}\right) \leq(1-\vartheta) \Phi\left(x_{0}\right)+\vartheta \Phi\left(x_{1}\right)-\frac{K}{2} \vartheta(1-\vartheta) \mathrm{d}^{2}\left(x_{0}, x_{1}\right) .
$$

Since $\left(\mathcal{P}_{2}(X), \mathrm{W}_{\mathrm{d}}\right)$ is a metric space, we can also consider geodesics at the level of probability measures: these are curves $\left(\mu_{\vartheta}\right)_{\vartheta \in[0,1]}$ in $\mathcal{P}_{2}(X)$ satisfying

$$
\mathrm{W}_{\mathrm{d}}\left(\mu_{\vartheta_{0}}, \mu_{\vartheta_{1}}\right)=\left(\vartheta_{1}-\vartheta_{0}\right) \mathrm{W}_{\mathrm{d}}\left(\mu_{0}, \mu_{1}\right), \quad 0 \leq \vartheta_{0} \leq \vartheta_{1} \leq 1 .
$$

In a pioneering paper, McCann [36] pointed out the role and the interest of geodesic convexity of suitable integral functionals in $\mathcal{P}_{2}(X)$ as the relative entropy,

$$
\operatorname{Ent}(\mu):= \begin{cases}\int_{X} \varrho \log \varrho \mathrm{dm} & \text { if } \mu=\varrho \mathfrak{m}, \varrho|\log \varrho| \in L^{1}(X, \mathrm{~m}) \\ +\infty & \text { otherwise. }\end{cases}
$$

As a beautiful example, Theorem 4 admits a nice reformulation in terms of its geodesic $K$-convexity.

Theorem 6 ([37, 18]). The Prékopa-Leindler inequality (7a, b) holds in the weighted Riemannian manifold $\left(\mathbb{M}, d_{g}, m\right)$ if and only if the functional Ent is geodesically $K$-convex in $\mathcal{P}_{2}(\mathbb{M})$. In this case, for every $\left(\mu_{\vartheta}\right)_{\vartheta \in[0,1]}$ satisfying (17), we have

$$
\begin{aligned}
\operatorname{Ent}\left(\mu_{\vartheta}\right) \leq(1-\vartheta) \operatorname{Ent}\left(\mu_{0}\right)+ & \vartheta \operatorname{Ent}\left(\mu_{1}\right) \\
& -\frac{K}{2} \vartheta(1-\vartheta) \mathrm{W}_{\mathrm{d}}\left(\mu_{0}, \mu_{1}\right) .
\end{aligned}
$$


Gradient flows in $\mathcal{P}_{2}(X)$ : convexity and contraction

The two remarkable properties highlighted by Theorems 5 and 6 can be better understood by the Jordan-Otto interpretation of the semigroup $\mathrm{P}^{*}$ as the gradient flow of the entropy functional in Wasserstein space $\mathcal{P}_{2}(\mathbb{M})$. There are (at least) three different ways to justify this interpretation. The first one combines the Benamou-Brenier result with the De Giorgi notion of curves of maximal slope [3], the second one is related to the so-called $\mathrm{JKO} /$ minimising movement variational scheme $[20,31,3]$ and the last one captures the energydistance interaction of convex functions in Euclidean space.

\section{Curves of maximal slope.}

One starts from the basic remark that solutions in $\mathbb{R}^{d}$ to the gradient flow equation

$$
\mathrm{x}^{\prime}(t)=-\nabla \Phi(\mathrm{x}(t))
$$

for a smooth function $\Phi: \mathbb{R}^{d} \rightarrow \mathbb{R}$ can be characterised by the maximal rate decay of $\Phi$, in the sense that along a general smooth curve $y$, we have

$$
\frac{\mathrm{d}}{\mathrm{d} t} \Phi(\mathrm{y}(t))=-\nabla \Phi(\mathrm{y}(t)) \cdot \mathrm{y}^{\prime}(t) \geq-|\nabla \Phi(\mathrm{y}(t))|\left|\mathrm{y}^{\prime}(t)\right|,
$$

whereas along any solution of (19), we have precisely

$$
\frac{\mathrm{d}}{\mathrm{d} t} \Phi(\mathrm{x}(t))=-\mid \nabla \Phi\left(\left.\mathrm{x}(t)|| \mathrm{x}^{\prime}(t)|=-| \nabla \Phi(\mathrm{x}(t))\right|^{2}=-\left|\mathrm{x}^{\prime}(t)\right|^{2},\right.
$$

an identity that is, in fact, equivalent to (19).

In order to mimic the above argument at the Wasserstein level, we first observe that, along any smooth solution $\mu_{t}=$ $\varrho_{t} \mathrm{~m}$ of the continuity equation (10b), it is possible to compute the time derivative of the entropy by an integration by parts, obtaining

$$
\begin{aligned}
\frac{\mathrm{d}}{\mathrm{d} t} \operatorname{Ent}\left(\mu_{t}\right) & =\int_{\mathbb{M}}\left\langle\nabla \log \varrho_{t}, \boldsymbol{v}_{t}\right\rangle \mathrm{d} \mu_{t} \\
& \geq-\left(\int_{\mathbb{M}}\left|\nabla \log \varrho_{t}\right|_{\mathrm{g}}^{2} \mathrm{~d} \mu_{t}\right)^{1 / 2}\left(\int_{\mathbb{M}}\left|\boldsymbol{v}_{t}\right|_{\mathrm{g}}^{2} \mathrm{~d} \mu_{t}\right)^{1 / 2} .
\end{aligned}
$$

Thus, the rate of decay of Ent is bounded from below by the product of the Wasserstein velocity of the curve (11) and the square root of the Fisher information functional

$$
\begin{aligned}
\mathrm{F}(\mu) & =\int_{\varrho>0}|\nabla \log \varrho|_{\mathrm{g}}^{2} \mathrm{~d} \mu_{t}=\int_{\varrho>0} \frac{|\nabla \varrho|_{\mathrm{g}}^{2}}{\varrho} \mathrm{d} \mathrm{m} \\
& =4 \int_{\mathbb{M}}|\nabla \sqrt{\varrho}|_{\mathrm{g}}^{2} \mathrm{~d} \mathrm{~m}, \quad \mu=\varrho \mathrm{m},
\end{aligned}
$$

which plays the same role as $|\nabla \Phi|$ in (20). On the other hand, along the solution $\mu_{t}=\mathrm{P}_{t}^{*} \mu=\left(\mathrm{P}_{t} \varrho\right) \mathrm{m}$ induced by (2), one has

$$
\frac{\mathrm{d}}{\mathrm{d} t} \operatorname{Ent}\left(\mu_{t}\right)=-\mathrm{F}\left(\mu_{t}\right)=-\left|\dot{\mu}_{t}\right|_{\mathrm{W}_{\mathrm{d}}}^{2},
$$

which corresponds to (21).

\section{The minimising movement approach.}

A second point of view (and, in fact, the original one adopted by Jordan-Kinderlehrer-Otto [31]) concerns a variational scheme that can be used to construct gradient flows in general metric spaces [20,3]. It is strongly related to the implicit Euler scheme for (19), which suggests approximating the values of the solution $\mathrm{x}$ at the discrete points $k \tau, k \in \mathbb{N}$, of a uniform grid of step $\tau>0$ by the solutions $\mathrm{X}_{\tau}^{k} \approx \mathrm{x}(k \tau)$ of the recursive discrete scheme

$$
\frac{1}{\tau}\left(\mathrm{X}_{\tau}^{k}-\mathrm{X}_{\tau}^{k-1}\right)+\nabla \Phi\left(\mathrm{X}_{\tau}^{k}\right)=0, \quad k=1,2, \cdots,
$$

starting from an approximation $\mathrm{X}_{\tau}^{0} \approx \mathrm{x}(0)$. One can select solutions to (24) by choosing $\mathrm{X}_{\tau}^{k}$ among the minimisers of

$$
\mathrm{X} \mapsto \frac{1}{2 \tau}\left|\mathrm{X}-\mathrm{X}_{\tau}^{k-1}\right|^{2}+\Phi(\mathrm{X})
$$

At the level of measures, replacing the Euclidean distance in $\mathbb{R}^{d}$ by the Wasserstein distance in $\mathcal{P}_{2}(X)$ and the function $\Phi$ by the relative entropy, one eventually obtains the following scheme.

Given $M_{\tau}^{0}:=\mu=\varrho m$, find $M_{\tau}^{k} \in \mathcal{P}_{2}(X)$ by minimising

$$
M \mapsto \frac{1}{2 \tau} \mathrm{W}_{\mathrm{d}}^{2}\left(M, M_{\tau}^{k-1}\right)+\operatorname{Ent}(M), \quad k=1,2, \cdots
$$

Defining $M_{\tau}(t)$ as the piecewise constant interpolant of the values $M_{\tau}^{k}$ in each interval $((k-1) \tau, k \tau]$, one can prove, under very general assumptions $[31,3,4]$, that the family $M_{\tau}$ converges locally uniformly in $\mathcal{P}_{2}(X)$ to the solution $\mu_{t}=\mathrm{P}_{t}^{*} \mu=$ $\left(\mathrm{P}_{t} \varrho\right) \mathrm{m}$ associated to $(2)$.

\section{Gradient flows and evolution variational inequalities.}

In the Euclidean framework, one can evaluate the derivative of the squared distance function of a solution $x$ of (19) from any given point $y \in \mathbb{R}^{d}$, obtaining

$$
\frac{\mathrm{d}}{\mathrm{d} t} \frac{1}{2}|\mathrm{x}(t)-y|^{2}=\langle\nabla \Phi(\mathrm{x}(t), y-\mathrm{x}(t)\rangle,
$$

and then use the $K$-convexity inequality

$$
\langle\nabla \Phi(x), y-x\rangle \leq \Phi(y)-\Phi(x)-\frac{K}{2}|x-y|^{2}
$$

to obtain the Evolution Variational Inequality $\left(\mathrm{EVI}_{K}\right)$

$$
\frac{\mathrm{d}}{\mathrm{d} t} \frac{1}{2}|\mathrm{x}(t)-y|^{2} \leq \Phi(y)-\Phi(\mathrm{x}(t))-\frac{K}{2}|\mathrm{x}(t)-y|^{2} \quad \forall y \in \mathbb{R}^{d} .
$$

It is not hard to see that (25), in fact, characterises the solutions to (19). Since (25) just involves the ambient distance and the driving functional $\Phi$, one is tempted to use it for a possible definition of gradient flows in arbitrary metric spaces [3].

Definition 7. Let $\left(\mathcal{X}, \mathrm{d}_{\mathcal{X}}\right)$ be a metric space and let $\Phi$ : $D(\Phi) \subset \mathcal{X} \rightarrow \mathbb{R}$ be a given 1.s.c. functional. A semigroup $\left(\mathrm{S}_{t}\right)_{t \geq 0}$ in $D(\Phi)$ is an $\mathrm{EVI}_{K}$-flow of $\Phi$ if, for every $x \in D(\Phi)$, the curve $\mathrm{x}_{t}:=\mathrm{S}_{t} x$ is locally Lipschitz in $(0, \infty)$ and solves

$$
\frac{\mathrm{d}}{\mathrm{d} t} \frac{1}{2} \mathrm{~d}_{\mathcal{X}}^{2}(\mathrm{x}(t), y) \leq \Phi(y)-\Phi(\mathrm{x}(t))-\frac{K}{2} \mathrm{~d}_{\mathcal{X}}^{2}(\mathrm{x}(t), y) \quad \forall y \in \mathcal{X}
$$


In general metric spaces, the existence of an $\mathrm{EVI}_{K}$-flow is a much stronger requirement than the simple energydissipation identity (21): it encodes both the $K$-convexity of $\Phi$ and a sort of infinitesimal Riemannian behaviour of the distance $d_{x}[19]$.

Theorem 8. Suppose that $\left(\mathrm{S}_{t}\right)_{t \geq 0}$ is an $\mathrm{EVI}_{K}$ flow for $\Phi$ on $\left(X, \mathrm{~d}_{X}\right)$. Then, $\mathrm{S}$ is a $K$-exponential contraction, i.e.

$$
\mathrm{d}_{\mathcal{X}}\left(\mathrm{S}_{t} x, \mathrm{~S}_{t} y\right) \leq \mathrm{e}^{-K t} \mathrm{~d}_{\mathcal{X}}(x, y) .
$$

If, moreover, any couple of points $x, y \in D(\Phi)$ can be joined by a geodesic in $\overline{D(\Phi)}$ then $\Phi$ is strongly $K$-geodesically convex.

Taking the above result and Otto heuristics concerning the Riemannian character of the Wasserstein distance into account, it is not completely surprising that Theorems 5 and 6 can be obtained as a consequence of the $\mathrm{EVI}_{K}$ characterisation of the Markov semigroup $\mathrm{P}^{*}$ in $\mathcal{P}_{2}(\mathbb{M})$. In fact, the weighted Riemannian manifold $\left(\mathbb{M}, d_{g}, m\right)$ satisfies the curvature-dimension $\mathrm{BE}(K, \infty)$ condition if and only if the semigroup $\left(\mathrm{P}_{t}^{*}\right)_{t \geq 0}$ is the $\mathrm{EVI}_{K}$ flow of the entropy functional in $\mathcal{P}_{2}(X)$ : for every $\mu \in D$ (Ent), the curve $\mu_{t}=\mathrm{P}_{t}^{*} \mu$ satisfies

$$
\frac{\mathrm{d}}{\mathrm{d} t} \frac{1}{2} \mathrm{~W}_{\mathrm{d}}^{2}\left(\mu_{t}, v\right) \leq \operatorname{Ent}(v)-\operatorname{Ent}\left(\mu_{t}\right)-\frac{K}{2} \mathrm{~W}_{\mathrm{d}}^{2}\left(\mu_{t}, v\right)
$$

for every $v \in D($ Ent $)$.

\section{$5 \quad$ Metric measure spaces, Gromov convergence and the Lott-Sturm-Villani curvature-dimension condition}

Let us now address the question of how to extend the previous results to a general metric measure space $(X, \mathrm{~d}, \mathrm{~m})$, i.e. a complete and separable metric space $(X, \mathrm{~d})$ endowed with a non-negative Borel measure $\mathrm{m}$, which we assume here to be with full support, satisfying the growth condition

$$
\mathrm{m}\left(B_{r}\left(x_{o}\right)\right) \leq a \mathrm{e}^{b r^{2}}, \quad a, b \in \mathbb{R}_{+} .
$$

This general class of spaces naturally arises when lower Ricci curvature bounds are considered, thanks to the following remarkable result of Gromov (see, for example, [30]) and to the deep contributions by Cheeger-Colding $[14,15,16]$.

Theorem 9 (Gromov compactness). Let $\left(\mathbb{M}^{h}, \mathrm{~d}_{\mathrm{g}}^{h}, \mathrm{~m}^{h}\right), h \in \mathbb{N}$, be a sequence of weighted Riemannian manifolds with uniformly bounded diameter and satisfying a uniform $\operatorname{BE}(K, N)$ condition, for some $K, N \in \mathbb{R}$ independent of $h$. Then, there exist a limit metric measure space $(X, d, m)$ and a subsequence $n \mapsto h(n)$ such that $\left(\mathbb{M}^{h(n)}, \mathrm{d}_{\mathrm{g}}^{h(n)}, \mathrm{m}^{h(n)}\right)$ converges to $(X, \mathrm{~d}, \mathrm{~m})$ under the measured Gromov convergence.

Perhaps the simplest way to introduce measured Gromov convergence for normalised (i.e. $\mathfrak{m}(X)=1$ ) metric measure spaces is to resort to another beautiful theorem of Gromov [30], characterising the equivalence class of metric measure spaces up to measure-preserving isometries. It relies on the notion of cylindrical metric functionals of the form

$$
\varphi^{\star}[(X, \mathrm{~d}, \mathrm{~m})]:=\int_{X^{n}} \varphi\left(\mathrm{d}\left(x_{i}, x_{j}\right)_{i, j=1}^{n}\right) \mathrm{d} \mathrm{m}^{\otimes n}\left(x_{1}, \cdots, x_{n}\right),
$$

where $n \in \mathbb{N}$ and $\varphi \in \mathrm{C}_{b}\left(\mathbb{R}^{n \times n}\right)$.

It is clear that if two metric measure spaces $\left(X_{i}, \mathrm{~d}_{i}, \mathrm{~m}_{i}\right), i=$ 1,2 , are isomorphic, i.e. there exists an isometry $\iota: X_{1} \rightarrow X_{2}$ preserving distances and volumes

$$
\mathrm{d}_{2}(\iota(x), \iota(y))=\mathrm{d}_{1}(x, y), \quad \mathrm{m}_{1}\left(\iota^{-1}(A)\right)=\mathrm{m}_{2}(A)
$$

for every couple of points $x, y \in X_{1}$ and every Borel set $A \subset X_{2}$, then $\varphi^{\star}\left[\left(X_{1}, \mathrm{~d}_{1}, \mathrm{~m}_{1}\right)\right]=\varphi^{\star}\left[\left(X_{1}, \mathrm{~d}_{2}, \mathrm{~m}_{2}\right)\right]$ for every cylindrical functional $\varphi^{\star}$ as in (28). The Gromov reconstruction theorem guarantees the converse property: if two normalised metric measure spaces are indistinguishable by all the cylindrical functionals then they are isomorphic. It justifies the following definition $[30,28]$ (see [40, 44, 27] for other equivalent approaches and for the relation with measured Gromov-Hausdorff convergence).

Definition 10 (Measured Gromov convergence). A sequence of normalised metric measure spaces $\left(X^{h}, \mathrm{~d}^{h}, \mathrm{~m}^{h}\right), h \in \mathbb{N}$, converges to a limit metric measure space $(X, \mathrm{~d}, \mathrm{~m})$ if, for every cylindrical functional $\varphi^{\star}$ as in (28), we have

$$
\lim _{h \rightarrow \infty} \varphi^{\star}\left[\left(X^{h}, \mathrm{~d}^{h}, \mathrm{~m}^{h}\right)\right]=\varphi^{\star}[(X, \mathrm{~d}, \mathrm{~m})] .
$$

In view of Theorem 9, it is natural to look for a synthetic definition of lower Ricci curvature bounds for general metric measure spaces that is stable under measured Gromov convergence, a programme that has been outlined in [14, Appendix 2]. Such a definition has been independently introduced by Sturm [40, 41] and Lott-Villani [35], starting from the smooth characterisation given by Theorem 6 .

Definition 11 (The Lott-Sturm-Villani $\mathrm{CD}(K, \infty)$ condition). A metric measure space $(X, \mathrm{~d}, \mathrm{~m})$ satisfies the $\mathrm{CD}(K, \infty)$ condition if the entropy functional Ent is geodesically $K$-convex in $\left(\mathcal{P}_{2}(X), \mathrm{W}_{\mathrm{d}}\right)$ : every couple $\mu_{0}, \mu_{1} \in D($ Ent $)$ can be connected by a geodesic $\left(\mu_{\vartheta}\right)_{\vartheta \in[0,1]}$ satisfying (17) along which (18) holds.

A similar but technically more complicated notion can be introduced in the case of a finite dimension upper bound $N<$ $\infty$ (and we do not distinguish here between $\mathrm{CD}, \mathrm{CD}^{*}$ or $\mathrm{CD}^{e}$ classes of spaces). Besides its intrinsic geometric structure, just involving the notion of distance (through Wasserstein geodesics) and measure (through the entropy functional), a crucial feature of the above definition is its stability with respect to measured Gromov convergence: if $\left(X^{h}, \mathrm{~d}^{h}, \mathrm{~m}^{h}\right), h \in$ $\mathbb{N}$, is a sequence of $\operatorname{CD}(K, N)$ metric measure spaces converging to $(X, \mathrm{~d}, \mathrm{~m})$ in the measured Gromov topology then $(X, \mathrm{~d}, \mathfrak{m})$ is a $\mathrm{CD}(K, N)$ metric measure space.

It is possible to prove that $\mathrm{CD}(K, N)$ metric measure spaces enjoy many of the geometric properties that are a consequence of the curvature-dimension condition in the smooth Riemannian setting (see the final remarks below).

Definition 11, however, captures only part of the information coded in the Riemannian formalism, since geodesic $K$ convexity of the entropy functional is also shared by Finsler (non-Riemannian) geometries: perhaps the simplest example is given by the space $\left(\mathbb{R}^{d},\|\cdot\|, \mathrm{Vol}\right)$, where the distance is induced by an arbitrary norm $\|\cdot\|$ (not necessarily Hilbertian) and the measure is the usual Lebesgue one. 
On the other hand, in many examples where a specific Dirichlet energy form and a corresponding Markov semigroup can be constructed, one may adopt the Bakry-Émery point of view to characterise a curvature-dimension condition. In this direction, Gromov wrote [29, page 85]: "There $i$ another option for the abstract theory of Ricci $\geq 0$, where instead of the metric one emphasizes the heat flow (diffusion), but at this stage it is unclear whether the two approaches are equivalent and if not which one is better for applications."

A relevant question is to single out a stronger condition for general metric measure spaces that is still stable under measured Gromov convergence and is capable of reproducing the pointwise gradient estimates (6) along a suitably adapted version of the heat flow. This would have the great advantage of combining both the tools from $\Gamma$-calculus and optimal transport and hopefully extending to the non-smooth framework many deep results and techniques available in the Riemannian setting. As explained in Section 4, the Wasserstein gradient flow of the entropy functional provides a unifying point of view for both the approaches and keeps the basic feature of a pure geometric formulation in terms of distance and measure.

\section{6 \\ $\operatorname{RCD}(K, \infty)$ spaces and the link between $\mathrm{BE}$ and $\mathrm{CD}$}

\section{The Cheeger energy and its $L^{2}$-gradient flow}

A first step in the direction of extending the Bakry-Émery approach to the setting of metric measure spaces concerns the construction of a canonical energy form, the so called Cheeger energy [13], and of the related evolution semigroup.

The Cheeger energy can be obtained by a relaxation procedure from the functional $\int_{X}|\nabla f|^{2}(x) \mathrm{d} m(x)$, initially defined on bounded Lipschitz functions and involving the local slope introduced in (12).

Definition 12 (The Cheeger energy). For every $f \in L^{2}(X, m)$, we define

$$
\begin{array}{r}
\operatorname{Ch}(f):=\inf \left\{\liminf _{n \rightarrow \infty} \frac{1}{2} \int_{X}\left|\nabla f_{n}\right|^{2} \mathrm{dm}:\right. \\
\left.f_{n} \in \operatorname{Lip}_{b}(X), f_{n} \stackrel{L^{2}}{\rightarrow} f\right\},
\end{array}
$$

with proper domain $D(\mathrm{Ch}):=\left\{f \in L^{2}(X, \mathrm{~m}): \mathrm{Ch}(f)<\infty\right\}$.

It is possible to prove that $\mathrm{Ch}$ is a convex, 2-homogeneous, lower semicontinuous functional in $L^{2}(X, m)$ with a dense domain. For every $f \in D(\mathrm{Ch})$, there exists at least one optimal sequence $\left(f_{n}\right)_{n} \subset \operatorname{Lip}_{b}(X)$ converging to $f$ in $L^{2}(X, \mathfrak{m})$ and realising the infimum in (29): the corresponding slopes $\left|\nabla f_{n}\right|$ converge strongly in $L^{2}(X, \mathfrak{m})$ to a unique limit that is called the weak gradient of $f$ and is denoted by $|\nabla f|_{w}$. The map $f \mapsto|\nabla f|_{w}$ is 1-homogeneous and subadditive, enjoys some natural calculus rules $[4,26]$ and represents $\mathrm{Ch}$ by the formula

$$
\mathrm{Ch}(f)=\frac{1}{2} \int_{X}|\nabla f|_{w}^{2} \mathrm{~d} \mathfrak{m}
$$

which can also be useful to define the Fisher information $\mathrm{F}(\mu)$ of a nonnegative measure $\mu=\varrho \mathrm{m}$ as in (22):

$$
\mathrm{F}(\mu)=8 \mathrm{Ch}(\sqrt{\varrho})=\int_{\varrho>0} \frac{|\nabla \varrho|_{w}^{2}}{\varrho} \mathrm{dm}, \quad \mu=\varrho \mathrm{m} .
$$

Even if $\mathrm{Ch}$ is not a quadratic form, it is still possible to use convex analysis to define the nonlinear Laplacian $-\Delta_{X} f$ as the element of minimal $L^{2}$-norm of its $L^{2}$-subdifferential, consisting of all the functions $\xi \in L^{2}(X, \mathfrak{m})$ satisfying the variational inequality

$$
\int_{X} \xi(g-f) \mathrm{dm} \leq \mathrm{Ch}(g)-\operatorname{Ch}(f) \quad \text { for every } g \in D(\mathrm{Ch}) .
$$

It is a remarkable result of the theory of gradient flows in Hilbert spaces that, for every $f \in L^{2}(X, \mathrm{~m})$, there exists a unique locally Lipschitz curve $\left(f_{t}\right)_{t>0}$ solving

$$
\frac{\mathrm{d}}{\mathrm{d} t} f_{t}=\Delta_{X} f_{t} \quad \text { for a.e. } t>0, \quad \lim _{t \downarrow 0} f_{t}=f .
$$

The map $\mathrm{P}_{t}: f \mapsto f_{t}$ defines a continuous semigroup of contractions in $L^{2}(X, \mathrm{~m})$; by the specific property of $\mathrm{Ch},\left(\mathrm{P}_{t}\right)_{t \geq 0}$ can also be extended to a semigroup of contractions in every $L^{p}(X, \mathfrak{m})$, preserving positivity, mass and constants.

It is then possible to prove, in many cases, that the semigroup $\left(\mathrm{P}_{t}\right)_{t \geq 0}$ coincides with the Wasserstein gradient flow of the entropy functional (as a curve of maximal slope and as a limit of the minimising movement variational scheme): this important identification holds, in particular, for the whole class of $\mathrm{CD}(K, \infty)$ metric measure spaces [24, 4].

Theorem 13. If $(X, \mathrm{~d}, \mathrm{~m})$ is a $\mathrm{CD}(K, \infty)$ space then, for every $\mu=\varrho \mathfrak{m} \in \mathcal{P}_{2}(X)$ with $\operatorname{Ent}(\mu)<\infty$, the curve $\mu_{t}=\left(\mathrm{P}_{t} \varrho\right) \mathrm{m}$ is locally Lipschitz in $\mathcal{P}_{2}(X)$ and the map $t \mapsto \operatorname{Ent}\left(\mu_{t}\right)$ is locally Lipschitz and satisfies (23); it is, moreover, the limit of the minimising movement scheme (30). Conversely, any locally Lipschitz curve $\mu_{t}=\varrho_{t} \mathrm{~m}, t \geq 0$, in $\mathcal{P}_{2}(X)$ solving (23) satisfies $\varrho_{t}=\mathrm{P}_{t} \varrho_{0}$.

\section{Quadratic Cheeger energies and a metric setting for the Bakry-Émery approach}

If one looks for a good metric framework where the BakryÉmery approach can be applied, there are at least two essential properties: the linearity of $\mathrm{P}_{t}$ and the link between distance and energy. Since $\mathrm{P}_{t}$ is originally defined as the gradient flow of the Cheeger energy in Hilbert space $L^{2}(X, \mathrm{~m})$, it is not surprising that the linearity of $\mathrm{P}_{t}$ is related to the quadraticity of $\mathrm{Ch}$; as a byproduct, it induces a nice connection between weak gradients and $\Gamma$-calculus.

Theorem 14 (Cheeger energy, Dirichlet forms and $\Gamma$ ). The semigroup $\left(\mathrm{P}_{t}\right)_{t \geq 0}$ is linear if and only if the Cheeger energy is quadratic, i.e. for every $f, g \in D(\mathrm{Ch})$,

$$
\mathrm{Ch}(f+g)+\operatorname{Ch}(f-g)=2 \mathrm{Ch}(f)+2 \mathrm{Ch}(g) .
$$

In this case, $\mathcal{E}(f, g):=\mathrm{Ch}(f+g)-\mathrm{Ch}(f)-\mathrm{Ch}(g)$ is a strongly local Dirichlet form, whose $\Gamma$-tensor coincides with the weak gradient and whose generator $\mathrm{L}$ coincides with $\Delta_{X}$ :

$$
\Gamma(f)=|\nabla f|_{w}^{2} \text { if } f \in D(\mathrm{Ch}), \quad \mathrm{L} f=\Delta_{X} f \text { if } f \in D\left(\Delta_{X}\right) .
$$


In the metric setting, a nice collection of smooth functions where the pointwise differential formulation of the BakryÉmery condition (5) can be stated is lacking; however, it is possible to give a suitable weak formulation that is still equivalent to the pointwise gradient estimate (6): e.g. the $\mathrm{BE}(K, \infty)$ condition is equivalent to asking that the map

$$
s \mapsto \mathrm{e}^{-2 K s} \int_{X} \Gamma\left(\mathrm{P}_{t-s} f\right) \mathrm{P}_{s} \phi \mathrm{dm}
$$

is increasing in $(0, t)$ for every $f \in D(\mathrm{Ch})$ and every nonnegative $\phi \in L^{\infty}(X, \mathfrak{m})$.

Concerning the link with distance, the very definition of Cheeger energy shows that every bounded $L$-Lipschitz function $f$ satisfies

$$
\Gamma(f)=|\nabla f|_{w}^{2} \leq L \quad \text { m-a.e. }
$$

In order to infer geometric properties on $(X, d)$ from the energy form, it is natural to ask that every function $f \in D(\mathrm{Ch})$ satisfying (33) admits an $L$-Lipschitz representative.

The $\operatorname{RCD}(K, \infty)$ condition and the entropic $\mathrm{EVI}_{K^{-}}$flow

Summarising the discussion above for a general metric measure space with a quadratic Cheeger energy, it is possible to ask for the Lott-Sturm-Villani $\mathrm{CD}(K, \infty)$ condition or for the Bakry-Émery condition $\operatorname{BE}(K, \infty)$. It turns out that these are, in fact, equivalent and can be unified by the notion of $\mathrm{EVI}_{K}$ flow $[4,5,6]$.

Theorem 15. For a general metric measure space $(X, \mathrm{~d}, \mathrm{~m})$, the following properties are equivalent:

(1) The Cheeger energy is quadratic according to (32) (and thus $\left(\mathrm{P}_{t}\right)_{t \geq 0}$ is linear) and $(X, \mathrm{~d}, \mathrm{~m})$ is a $\operatorname{CD}(K, \infty)$ space.

(2) The Cheeger energy is quadratic according to (32), every function satisfying (33) is L-Lipschitz and the BakryÉmery condition holds (in a suitably weak formulation).

(3) The entropy functional Ent admits a $\mathrm{EVI}_{K}$ flow according to Definition (7).

This result leads to the following definition.

Definition 16 (The Riemannian curvature-dimension condition). A metric measure space $(X, \mathrm{~d}, \mathfrak{m})$ satisfies the $\operatorname{RCD}(K, \infty)$ condition if one of the equivalent properties of Theorem 15 is satisfied.

The theorem above has been remarkably extended to the case of the finite dimension condition by Erbar-KuwadaSturm [21] by introducing a suitable notion of $\mathrm{EVI}_{K, N}$ flow for the entropy power functional

$$
\mathrm{H}_{N}(\mu):=\exp \left(-\frac{1}{N} \operatorname{Ent}(\mu)\right)
$$

A different approach, using Rény entropies in the original formulation of the Lott-Sturm-Villani condition, has also been developed by [1]. A crucial result due to the formulation in terms of entropy and Wasserstein distance is the following stability property with respect to measured Gromov convergence $[6,27]$.
Theorem 17. If $\left(X^{h}, \mathrm{~d}^{h}, \mathrm{~m}^{h}\right), h \in \mathbb{N}$, is a sequence of $\operatorname{RCD}(K, N)$ metric measure spaces converging to $(X, \mathrm{~d}, \mathrm{~m})$ in the measured Gromov topology then $(X, \mathrm{~d}, \mathrm{~m})$ is an $\operatorname{RCD}(K, N)$ metric measure space. Moreover, if the diameters of $X^{h}$ are uniformly bounded and $\lambda_{k}\left(\mathrm{~L}^{h}\right), k \in \mathbb{N}$, are the ordered sequences of eigenvalues of the compact operator $\mathrm{L}^{h}$, we have

$$
\lim _{h \rightarrow \infty} \lambda_{k}\left(\mathrm{~L}^{h}\right)=\lambda_{k}(\mathrm{~L}) \quad \text { for every } k \in \mathbb{N} \text {. }
$$

\section{$7 \quad$ Applications}

It is really difficult to give even a partial account of the ongoing and striking developments of the metric theory of CD and RCD spaces. Both are sufficiently flexible and strong to guarantee a series of structural geometric results: among them, we quote the tensorisation property, the global-to-local and localto-global characterisations of the $\mathrm{CD} / \mathrm{RCD}$ conditions and the development of a nice first and second order calculus [26].

We now recall some of the most important geometric and functional analytic estimates (often stated in particular exemplifying cases) that can be derived for a general metric measure space $(X, \mathrm{~d}, \mathrm{~m})$. We start from the properties valid for all $\mathrm{CD}(K, N)$ spaces, where the recent results of CavallettiMondino $[11,12]$ solve a series of important open problems and show the power of the optimal transport approach (in the RCD framework, they can also be deduced by $\Gamma$-calculus tools - see [9]).

Bishop-Gromov inequality: For $x_{0} \in X$, the map

$$
r \mapsto \frac{\mathrm{m}\left(B_{r}\left(x_{0}\right)\right)}{\int_{0}^{r} s^{K, N}(t) \mathrm{d} t} \quad \text { is nonincreasing, }
$$

where $s^{K, N}$ is the function providing the measure of the spheres in the model space of Ricci curvature $K$ and dimension $N$ [44].

Bonnet-Myers diameter estimate: If $K>0$ then the diameter of $X$ is bounded by $\pi \sqrt{(N-1) / K}$.

Spectral gap and Poincaré inequality: If $K>0$ then

$$
\int_{X}(f-\bar{f})^{2} \mathrm{~d} \mathfrak{m} \leq \frac{N-1}{N K} \int_{X}|\nabla f|_{w}^{2} \mathrm{~d} \mathfrak{m}, \quad \bar{f}=\int_{X} f \mathrm{~d} \mathfrak{m},
$$

and a sharp inequality also holds for $L^{p}$ with $p \neq 2$ [12].

Log-Sobolev and Talagrand inequalities: If $K>0$ and $\mathfrak{m}(X)=1$ then [12]

$$
\frac{K N}{2(N-1)} \mathrm{W}_{\mathrm{d}}^{2}(\mu, \mathrm{m}) \leq \operatorname{Ent}(\mu) \leq \frac{N-1}{2 K N} \mathrm{~F}(\mu) .
$$

Sharp Sobolev inequalities: If $K>0, N>2,2<p \leq 2^{\star}:=$ $2 N /(N-2)$ then [12]

$$
\|f\|_{L^{p}}^{2} \leq\|f\|_{L^{2}}^{2}+\frac{(p-2)(N-1)}{K N} \int_{X}|\nabla f|_{w}^{2} \mathrm{~d} \mathrm{~m} .
$$

Levy-Gromov inequality: If $m(X)=1, \operatorname{diam}(X)=D$ and $A \subset X$ with perimeter $P(A)<\infty$ then

$$
P(A) \geq \mathcal{I}_{K, N, D}(\mathfrak{m}(A)),
$$


where $\mathcal{I}$ is a suitably defined model isoperimetric profile for the parameters $K, N, D$ (such as the $N$-dimensional sphere, when $N$ is an integer and $K>0$ ). The case $N=\infty$ holds in RCD spaces [9, Cor. 8.5.5], [7].

Let us now consider the specific case of $\operatorname{RCD}(K, N)$ spaces, where $\left(\mathrm{P}_{t}\right)_{t \geq 0}$ is a linear Markov semigroup associated to a Markov process and second order calculus tools can also be developed [22].

Li-Yau and Harnack inequalities: If $K \geq 0$ and $N<\infty$ then [9, Cor. 6.7.6]

$$
\begin{gathered}
\mathrm{L}\left(\log \mathrm{P}_{t} f\right) \geq-\frac{N}{2 t} \quad t>0 \\
\mathrm{P}_{t} f(x) \leq \mathrm{P}_{t+s} f(y)\left(\frac{t+s}{t}\right)^{N / 2} \mathrm{e}^{\mathrm{d}^{2}(x, y) / 2} .
\end{gathered}
$$

The splitting theorem [25]: If $K \geq 0, N \in[2, \infty)$ and $X$ contains a line, i.e. there exists a map $\gamma: \mathbb{R} \rightarrow X$ such that $\mathrm{d}(\gamma(s), \gamma(t))=|t-s|$ for every $s, t \in \mathbb{R}$, then $(X, \mathrm{~d}, \mathfrak{m})$ is isomorphic to the product of $\mathbb{R}$ (with Euclidean distance and the usual Lebesgue measure) and a $\operatorname{RCD}(0, N-1)$ space.

The maximal diameter theorem [32]: If $(X, \mathrm{~d}, \mathrm{~m})$ satisfies the $\operatorname{RCD}(N, N+1)$ condition with $N>0$ and there exists points $x, y \in X$ such that $\mathrm{d}(x, y)=\pi$ then $(X, \mathrm{~d}, \mathrm{~m})$ is isomorphic to the spherical product of $[0, \pi]$ and a $\operatorname{RCD}(N-1, N)$ space with diameter less than $\pi$.

Volume-to-metric cones [23]: If $K=0$, there exists $x_{o} \in X$ such that $\mathrm{m}\left(B_{r}\left(x_{o}\right)\right)=(R / r)^{N} \mathrm{~m}\left(B_{r}\left(x_{o}\right)\right)$ for some $R>r>0$ and the sphere centred at $x_{0}$ of radius $R / 2$ contains at least three points then the ball $B_{R}\left(x_{o}\right)$ is locally isometric to the ball $B_{R}\left(y_{o}\right)$ of the cone $Y$ built over an $\operatorname{RCD}(N-2, N-1)$ space.

We conclude this brief review by noting that there have been some recent striking applications to time-dependent metric measure spaces and Ricci flows [43, 33].

\section{Bibliography}

[1] L. Ambrosio, A. Mondino, and G. Savaré. Nonlinear diffusion equations and curvature conditions in metric measure spaces. ArXiv e-prints, 1509.07273, 2015.

[2] L. Ambrosio, M. Erbar, and G. Savaré. Optimal transport, Cheeger energies and contractivity of dynamic transport distances in extended spaces. Nonlinear Anal., 137:77-134, 2016.

[3] L. Ambrosio, N. Gigli, and G. Savaré. Gradient flows in metric spaces and in the space of probability measures. Lectures in Mathematics ETH Zürich. Birkhäuser Verlag, Basel, second edition, 2008.

[4] L. Ambrosio, N. Gigli, and G. Savaré. Calculus and heat flow in metric measure spaces and applications to spaces with Ricci bounds from below. Invent. Math., 195(2):289-391, 2014.

[5] L. Ambrosio, N. Gigli, and G. Savaré. Metric measure spaces with Riemannian Ricci curvature bounded from below. Duke Math. J., 163(7):1405-1490, 2014.

[6] L. Ambrosio, N. Gigli, and G. Savaré. Bakry-Émery curvature-dimension condition and Riemannian Ricci curvature bounds. Ann. Probab., 43(1):339-404, 2015.

[7] L. Ambrosio and A. Mondino. Gaussian-type isoperimetric inequalities in $\operatorname{RCD}(K, \infty)$ probability spaces for positive $K$. Atti Accad. Naz. Lincei Rend. Lincei Mat. Appl., 27(4):497514, 2016.
[8] D. Bakry and M. Émery. Diffusions hypercontractives. In Séminaire de probabilités, XIX, 1983/84, volume 1123, pages 177-206. Springer, Berlin, 1985.

[9] D. Bakry, I. Gentil, and M. Ledoux. On Harnack inequalities and optimal transportation. Ann. Sc. Norm. Super. Pisa Cl. Sci. (5), 14(3):705-727, 2015.

[10] J.-D. Benamou and Y. Brenier. A computational fluid mechanics solution to the Monge-Kantorovich mass transfer problem. Numer. Math., 84(3):375-393, 2000.

[11] F. Cavalletti and A. Mondino. Sharp and rigid isoperimetric inequalities in metric-measure spaces with lower Ricci curvature bounds. To appear in Invent. Math., arXiv:1502.06465, 2015.

[12] F. Cavalletti and A. Mondino. Sharp geometric and functional inequalities in metric measure spaces with lower Ricci curvature bounds. To appear in Geom. Topol., ArXiv eprints:1505.02061, 2015.

[13] J. Cheeger. Differentiability of Lipschitz functions on metric measure spaces. Geom. Funct. Anal., 9(3):428-517, 1999.

[14] J. Cheeger and T. H. Colding. On the structure of spaces with Ricci curvature bounded below. I. J. Differential Geom., 46(3):406-480, 1997.

[15] J. Cheeger and T. H. Colding. On the structure of spaces with Ricci curvature bounded below. II. J. Differential Geom., 54(1):13-35, 2000.

[16] J. Cheeger and T. H. Colding. On the structure of spaces with Ricci curvature bounded below. III. J. Differential Geom., 54(1):37-74, 2000

[17] D. Cordero-Erausquin, R. J. McCann, and M. Schmuckenschläger. A Riemannian interpolation inequality à la Borell, Brascamp and Lieb. Invent. Math., 146(2):219-257, 2001.

[18] D. Cordero-Erausquin, R. J. McCann, and M. Schmuckenschläger. Prékopa-Leindler type inequalities on Riemannian manifolds, Jacobi fields, and optimal transport. Ann. Fac. Sci. Toulouse Math. (6), 15(4):613-635, 2006.

[19] S. Daneri and G. Savaré. Eulerian calculus for the displacement convexity in the Wasserstein distance. SIAM J. Math. Anal., 40(3):1104-1122, 2008.

[20] E. De Giorgi. New problems on minimizing movements. In C. Baiocchi and J. L. Lions, editors, Boundary Value Problems for PDE and Applications, pages 81-98. Masson, 1993.

[21] M. Erbar, K. Kuwada, and K.-T. Sturm. On the equivalence of the entropic curvature-dimension condition and Bochner's inequality on metric measure spaces. Invent. Math., 201(3):9931071, 2015

[22] N. Gigli. Nonsmooth differential geometry - An approach tailored for spaces with Ricci curvature bounded from below. To Mem. Amer. Math. Soc., ArXiv:1407.0809, 2015.

[23] N. Gigli and G. de Philippis. From volume cone to metric cone in the nonsmooth setting. Geom. Funct. Anal., 26(6):15261587,2016

[24] N. Gigli, K. Kuwada, and S. Ohta. Heat flow on Alexandrov spaces. Comm. Pure Appl. Math., 66:307-331, 2013.

[25] N. Gigli. An overview of the proof of the splitting theorem in spaces with non-negative Ricci curvature. Anal. Geom. Metr. Spaces, 2:169-213, 2014.

[26] N. Gigli. On the differential structure of metric measure spaces and applications. Mem. Amer. Math. Soc., 236(1113):vi+91, 2015.

[27] N. Gigli, A. Mondino, and G. Savaré. Convergence of pointed non-compact metric measure spaces and stability of Ricci curvature bounds and heat flows. Proc. Lond. Math. Soc. (3), 111(5):1071-1129, 2015.

[28] A. Greven, P. Pfaffelhuber, and A. Winter. Convergence in distribution of random metric measure spaces $(\Lambda$-coalescent measure trees). Probab. Theory Related Fields, 145(1-2):285$322,2009$. 
[29] M. Gromov. Sign and geometric meaning of curvature. Rend. Sem. Mat. Fis. Milano, 61:9-123 (1994), 1991.

[30] M. Gromov. Metric structures for Riemannian and nonRiemannian spaces. Modern Birkhäuser Classics. Birkhäuser, Boston, 2007.

[31] R. Jordan, D. Kinderlehrer, and F. Otto. The variational formulation of the Fokker-Planck equation. SIAM J. Math. Anal., 29(1):1-17, 1998.

[32] C. Ketterer. Cones over metric measure spaces and the maximal diameter theorem. J. Math. Pures Appl. (9), 103(5):12281275, 2015.

[33] E. Kopfer and K.-T. Sturm. Heat flows on time-dependent metric measure spaces and super-Ricci flows. ArXiv e-prints, 2016.

[34] K. Kuwada. Duality on gradient estimates and Wasserstein controls. J. Funct. Anal., 258(11):3758-3774, 2010.

[35] J. Lott and C. Villani. Ricci curvature for metric-measure spaces via optimal transport. Ann. of Math. (2), 169(3):903991, 2009.

[36] R. J. McCann. A convexity principle for interacting gases. Adv. Math., 128(1):153-179, 1997.

[37] F. Otto and C. Villani. Generalization of an inequality by Talagrand and links with the logarithmic Sobolev inequality. $J$. Funct. Anal., 173(2):361-400, 2000.

[38] F. Otto and M. Westdickenberg. Eulerian calculus for the contraction in the Wasserstein distance. SIAM J. Math. Anal., 37(4):1227-1255 (electronic), 2005.

[39] F. Santambrogio. Optimal transport for applied mathematicians, volume 87 of Progress in Nonlinear Differential Equations and their Applications. Birkhäuser/Springer, Cham, 2015. Calculus of variations, PDEs, and modeling.

[40] K.-T. Sturm. On the geometry of metric measure spaces. I. Acta Math., 196(1):65-131, 2006.

[41] K.-T. Sturm. On the geometry of metric measure spaces. II. Acta Math., 196(1):133-177, 2006.

[42] K.-T. Sturm and M.-K. von Renesse. Transport inequalities, gradient estimates, entropy, and Ricci curvature. Comm. Pure Appl. Math., 58(7):923-940, 2005.
[43] K. Sturm. Super-Ricci flows for metric measure spaces. I. arXiv:1603.02193, 2016.

[44] C. Villani. Optimal transport. Old and new, Volume 338 of Grundlehren der Mathematischen Wissenschaften. SpringerVerlag, Berlin, 2009.

[45] F.-Y. Wang. Equivalent semigroup properties for the curvature-dimension condition. Bull. Sci. Math., 135(67):803-815, 2011.

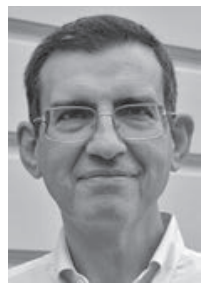

Luigi Ambrosio [luigi.ambrosio@sns.it] is a professor of mathematics at Scuola Normale Superiore, Pisa. His research interests include geometric measure theory, calculus of variations and optimal transport.

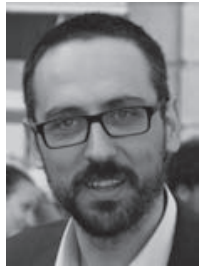

Nicola Gigli[ngigli@sissa.it] is a professor of mathematics at SISSA, Trieste. He is currently interested in geometric and non-smooth analysis, with a particular focus on properties of spaces with curvature bounded from below.

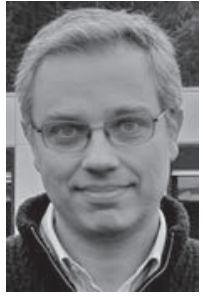

Giuseppe Savaré [giuseppe.savare@unipv. it] is a professor of mathematics at Pavia University. His current research interests involve optimal entropy transport problems and variational methods for gradient flows and rate-independent evolutions. This article follows his invited talk at the 7th European Congress of Mathematics in Berlin, 2016. 